\title{
Transmit Antenna Selection Strategies for SC- FDMA- IDMA Massive MIMO Systems
}

\author{
Roopali Agarwal", M. Shukla \\ Department of Electronics Engineering, Harcourt Butler Technical University, Kanpur, India
}

Received March 17, 2020; Revised April 13, 2020; Accepted April 27, 2020

Copyright $\odot 2020$ by authors, all rights reserved. Authors agree that this article remains permanently open access under the terms of the Creative Commons Attribution License 4.0 International License

\begin{abstract}
The $5 \mathrm{G}$ wireless offers many advancements over the prevalent 4 G LTE communication networks, such as enhanced data transmission rates (in order of Gbps), Substantially reduced latency, many times increase in the Base Station Capacity and praiseworthy betterments in the QoS offered to the users. IDMA has already proven its potential in $5 \mathrm{G}$ and it can be easily integrated in to massive MIMO systems. The ideology behind the concept of Massive MIMO is to match the requirement for efficient usage of spectrum, and is implemented using several numbers of antennas at the Base Station, catering to a number of subscribers concurrently using same band of the given frequency. However, the cost and complexity of implementation of such large-scale antenna systems is quite high. Thus for reduction in the number of radio frequency chains, the technique of Transmit Antenna Selection is used. In this paper, we propose two transmit antenna selection strategies considered for individual user or overall users based on selection criteria maximum sum rate or minimum bit error performance considering for (MIMO) multiuser multiple input and multiple output single carrier frequency division multiple access based interleave division multiple access wireless system (MIMO-SCFDMA-IDMA). We have taken this scheme for the uplink communication and selection of the subcarrier is through the bulk selection. The performance may get degraded in case of heavy load of multiple subcarriers attached to one antenna, which will in turn call for the role of power amplifier and thus efficiency of the system may go down. Therefore, here we prefer bulk subcarrier selection instead of per subcarrier selection. It is shown in this paper that enhanced sum rate can be achieved as more users are allowed to transmit concurrently, and thus multiuser gain is achieved. We also demonstrated the comparison with simulation result of sum rate performance and Bit error rate performance of varying users. Result shows that antenna selection based on overall system is better than the antenna selection considering each user.
\end{abstract}

Keywords Antenna Selection, Large MIMO, SC-FDMA, IDMA, MMSE Detection, Interleaver

\section{Introduction}

Multiple input multiple output (MIMO) systems are necessary part of various wireless standards such as $4 \mathrm{G}$, WiMax, 802.11n, HSPA+.MIMO is that the technique to transfer the information independently from over one antenna at the same time, and at receiver end information is received by one or more antenna, without additional bandwidth or transmit power, better performance and higher data rates can be obtained in MIMO system, but the eminent problem of MIMO systems arises due to the increase in complexness and cost induced by RF chain [1].

Antenna selection is a robust technique for providing low complexity to the system and aggrandizing the performance. Low complexity with low cost of the system is brought about by reducing the number of RF chains in spite of using complete RF chains.

As compared to the OFDMA techniques, the peak to average power ratio is less in the SC-FDMA scheme, and due to this quality, it finds its suitability in the third generation partnership project (3GPP) long-term evolution (LTE) and in the Fifth generation uplink communications also.

In communication systems, the noise and distortion of the signal due to the impairment in the channel may cause burst errors were not only discrete bits become in error but also a consequent group of bits. To combat such type of errors, the interleaveres are introduced in the system where the bits are reordered at the transmitter to spread the burst error when it occurs and converting them from burst error to discrete errors. At the receiver end, the interleaved information is organized back to the initial sequence with 
the assistance of de-interleaver [14]. As a result of interleaving, related to noise within the transmission seems to be statistically independent at the receiver and therefore permits higher error correction. The interleaver based multiple-access scheme [13] has been studied for high spectral efficiency, improved performance and low receiver complexity. For efficient communication there's demand of high spectral efficiency i.e. high data rate and low fading thus MIMO-SC-FDMA scheme with IDMA has been employed in the system.

Diversity mechanism, in communication system, is well known technique for improving the BER performance of communication receivers which improve the performance of wireless link at a comparatively low price. They're utilized to primarily improve performance over a fading radio channel. In general, fading is classed into small scale and large scale fading. This notion is additionally associated with coherence time of the channel that measures the time over that fading method is absolutely related to. In general, the fading is claimed to be slow if the symbol period is less than the coherence time of the channel, otherwise it's thought of to be fast fading. In slow fading, specific fade can have an effect on serial symbols whereas in fast fading the fading is not dependent from symbol to symbol. The number of the transmitting and receiving antenna systems decide the order of diversity. As there are $\mathrm{M}$ transmitting antennas and correspondingly, $\mathrm{N}$ receiving antennas, the diversity is achieved of the order $\mathrm{M} * \mathrm{~N}$, and as the diversity order increases it gives better bit error performance.

In this paper, transmit antenna selection is applied to MIMO SC-FDMA-IDMA system through bulk selection (choosing same antennas for all subcarriers), for improving the bit error (BER) and sum-rate performance of the system presented in this paper. The selection criteria considered are two: sum rate be maximized and bit error rate [2] be minimized, and Antenna selection strategy is proposed based on antenna selection at each user separately and at all users jointly. There are many receivers to support MIMO system such as Zero forcing (ZF) receiver, maximum likelihood (ML) receivers and minimum mean squared error (MMSE) are the receivers used for supporting MIMO systems.

Due to simplicity and single tap filters in the frequency domain, on LTE uplink systems, ZF-FDE or MMSE-FDE, the linear frequency domain equalization (FDE) is used. In this work, the MMSE detection has been employed in the system due to its better demonstrated performance observed from the literature.

The organization of the paper is as follows: In the section 2, MIMO SC-FDMA-IDMA system model is described. In section 3 and 4 antenna selection criterion and various antennas selection strategy scheme is discussed, in next section, the simulation result of sum rate and error rate performance has been presented. Finally, the section 6 concludes the paper.

\section{MIMO-SC-FDMA-IDMA SYSTEM MODEL}

Initially, large scale multiuser SC-FDMA-IDMA based MIMO uplink as shown in figurel has been considered with $\mathrm{N}_{\mathrm{r}}$ receive antennas at the base station communicating with $\mathrm{U}$ users where $\left(\mathrm{U}^{*} \mathrm{~N}_{\mathrm{t}} \leq \mathrm{N}_{\mathrm{r}}\right)$. Each user has $\mathrm{N}_{\mathrm{t}}$ antennas for transmission and $\mathrm{N}_{\mathrm{c}}$ subcarriers. Let the data stream of user-i be $d_{i}$. A forward error correction (FEC) code is used to encode this data stream, generating a chip sequence $C_{i}$ Here, the spreaded data is denoted by "chip" instead of "bit" as the FEC encoding may include spreading or repetition coding. Then $\mathrm{C}_{\mathrm{i}}$ is permutated by a user-specific interleaver-i to produce chip sequence $V_{i}$ and then, map the coded bit stream to time-domain constellation points in the finite average transmit power Es per symbol. An n-point discrete Fourier transform (DFT) is used to perform modulation of these $\mathrm{n}$ time-domain symbols on to orthogonal frequency bands [3]. The time-domain constellation points for the $i$ th user are subsumed in the vector $\mathrm{x}_{(\mathrm{i})}{ }^{(\mathrm{I})}=\left[\mathrm{x}_{1}{ }^{(\mathrm{i})}, \mathrm{x}_{2}{ }^{(\mathrm{i})}, \mathrm{x}_{3}{ }^{(\mathrm{i})} \ldots \ldots, \mathrm{x}_{\mathrm{n}}{ }^{(\mathrm{i})}\right]$. The output of the DFT block vectors ${ }^{(\mathrm{i})}=\left[\mathrm{s}_{1}{ }^{(\mathrm{i})}, \mathrm{s}_{2}{ }^{(\mathrm{i})}, \mathrm{s}_{3}{ }^{(\mathrm{i})} \ldots . ., \mathrm{s}_{\mathrm{n}}{ }^{(\mathrm{i})}\right]^{\mathrm{T}}$, namely the frequency-domain symbol [4]. Specifically, for each user, the frequency-domain symbols are first mapped to data-carrying subcarriers and then, transformed back to the time domain with an inverse DFT (IDFT). After getting the subset that achieve the antenna selection criteria goal, all U users transmit their time-domain signals simultaneously over the wireless channel.
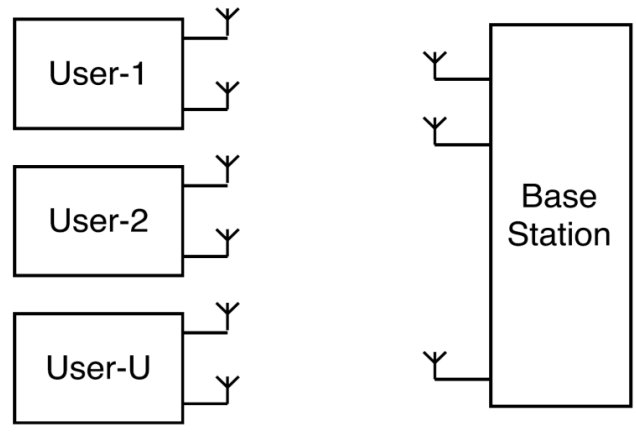

Figure 1. Multiuser MIMO Uplink System

At the base station time domain signals is received at each receive antenna, and transformed back into frequency domain using DFT. The input-output relationship on the wth subcarrier in the frequency domain can be mathematically Modeled as $y_{w}=H_{s w} s_{w}+n_{w}$

$$
\begin{gathered}
y_{w}=\left[\begin{array}{c}
y_{w}^{(1)} \\
\vdots \\
y_{w}^{(N r)}
\end{array}\right], H_{S w}=\left[\begin{array}{ccc}
h_{w}^{(1,1)} & \ldots & h_{w}^{\left(1, N_{S} U\right)} \\
\vdots & \ddots & \vdots \\
h_{w}^{(N r, 1)} & \cdots & h_{w}^{\left(N r, N_{S} U\right)}
\end{array}\right] \\
s_{w}=\left[s_{w}^{(1)}, s_{w}^{(2)} \ldots \ldots \ldots s_{w}^{\left(N_{S} U\right)}\right]^{T}
\end{gathered}
$$

and

$$
n_{w}=\left[n_{w}^{(1)}, n_{w}^{(2)}, \ldots \ldots, n_{w}^{\left(N_{r}\right)}\right]^{T}
$$


Here $\mathrm{y}_{\mathrm{w}}{ }^{(\mathrm{I})}$ is the frequency domain received symbol on the $\mathrm{w}^{\text {th }}$ subcarrier for the $\mathrm{i}^{\text {th }}$ receive antenna. The $\left(\mathrm{N}_{\mathrm{r}} \mathrm{XN}_{\mathrm{s}} \mathrm{U}\right)$ matrix $\mathrm{H}_{\mathrm{sw}}$ contains the channel gains between the $\mathrm{N}_{\mathrm{r}}$ receive antennas and selected $\left(\mathrm{N}_{\mathrm{S}} \mathrm{XU}\right)$ transmit antenna, so $\mathrm{H}_{\mathrm{sw}}$ is created by selecting $\left(\mathrm{N}_{\mathrm{s}} \mathrm{XU}\right)$ columns from $\mathrm{N}_{\mathrm{t}} \mathrm{XU}$ columns of $\mathrm{H}$ matrix for the wth subcarrier. $\mathrm{h}^{\mathrm{ij}}$ is modeled as independent complex Gaussian random variable with zero mean and variance as unity, $\mathrm{n}_{\mathrm{w}}$ denote the additive white Gaussian noise with i.i.d. entries $\mathrm{n}_{\mathrm{w}}{ }^{(\mathrm{i})} \sim \mathrm{N}\left(0, \sigma^{2}\right)$, where $\sigma^{2}$ is the variance of noise. The scalar $\mathrm{s}_{\mathrm{w}}{ }^{(\mathrm{j})}$ denotes the transmitted symbol by the jth user on the wth subcarrier, the equalized symbols $\mathrm{s}_{\mathrm{w}}=\mathrm{W}_{\mathrm{w}} \mathrm{y}_{\mathrm{w}}$ with the per subcarrier equalization matrix defined as [5].

$$
W_{w}=\left(H_{w}^{H} H_{w}+N_{0} E_{s}^{-1} I_{N_{S} U}\right)^{-1} H_{w}^{H}
$$

And then adopting an iterative sub-optimal receiver structure, consisting of the primary signal estimator (PSE) and $U$ users are simultaneously transmitting and posteriori probability (APP) decoders (DECs) are soft in and soft out component, they iteratively update information by computing log-likelihood ratios (LLRs) the data is iterated for pre-decided number of iterations before finally taking hard decision on it.

\section{Antenna Selection Criteria for MIMO-SC-FDMA-IDMA Uplink System}

The antenna selection criteria is based on the goal of providing maximum sum rate or/and minimum bit error rate criteria. In this paper, perfect channel information is considered to be available at both transmitter and receiver ends.

\subsection{Max-Sum Rate Selection Criteria}

In this paper, both the strategies on the maximum sum rate criteria have been applied for simulation purposes. The goal of transmit antenna subset selection is to achieve the maximum sum rate. It is mentioned here again that all subcarrier uses same subset of antenna in bulk selection process.

The achievable sum rate of MMSE receivers can be written as [5-6]

$$
E\left(R_{S C-M M S E}\right)=\sum_{i=1}^{N_{t}} E\left(\log \left(1+\operatorname{SIN} R_{i}\right)\right)
$$

By using Jensen's inequality, the lower bound of

$$
\begin{gathered}
E\left(R_{S C-M M S E}\right) \geq-\sum_{i=1}^{N-1} \log \left(\frac { 1 } { \mathrm { N } } \sum _ { k = 0 } ^ { N - 1 } E \left(\left[\left(\frac{1}{N_{t} \sigma^{2}} H_{k}^{H} H_{k}+\right.\right.\right.\right. \\
\left.\left.\left.I)^{-1}\right]_{i, i}\right)\right)
\end{gathered}
$$

\subsection{Min-Error Rate Selection Criteria}

It can be demonstrated from the analysis and further simulation that error rate can be minimized by maximizing the SINR. Therefore, the goal of these selection criteria is to maximize the SINR of the received signals in the presented system.

For MMSE receivers, the output SINR of the $i^{\text {th }}$ symbol for each subcarrier is given by-

$$
\operatorname{SINR}_{i}=\frac{1}{\left[\frac{1}{\mathrm{~N}} \sum_{\mathrm{k}=0}^{\mathrm{N}-1}\left(\frac{1}{N_{t} \sigma^{2}} H_{k}^{H} H_{k}+I\right)^{-1}\right]_{(i, i)}}
$$

\section{Antenna Selection Strategy for MIMO-SC-FDMA-IDMA Uplink System}

In this section, two Antenna selection strategies is proposed and simulation results presents that the antenna selection is another economical way to get diversity over MIMO channel on the basis of two selection criteria which is explained in section II.

\subsection{Antenna Selection Based on Each User Separately}

In this paper, it has been considered that transmit antenna selection $\mathrm{N}_{\mathrm{s}}$ antennas selected for transmission per user that satisfy the selection criteria, $\mathrm{Hij}^{\mathrm{n}} \in \mathrm{C}^{\mathrm{Nr} * \mathrm{NsU}}$ is the flat frequency domain channel matrix between the jth transmit antenna and ith receive antenna over the nth subcarrier.

In the strategy of antenna selection, a subset of transmit antenna $\mathrm{N}_{\mathrm{s}}$ is selected out of $\mathrm{N}_{t} \alpha_{\mathrm{K}}{ }^{\mathrm{u}}$ is the kth subset of the all possible combination of the antennas at the uth user, where $\alpha_{\mathrm{K}}{ }^{\mathrm{u}}$ can be as

$$
\alpha_{k}^{u}=\left\{I_{t}\right\}_{t=1}^{N_{t}}
$$

where $\mathrm{I}_{\mathrm{t}}$ represents the th transmit antenna.

Therefore, it is set to 0 if and only if the th transmit antenna is not active, and otherwise 1 .

$$
A=\left(\begin{array}{c}
N_{t} \\
N_{s}
\end{array}\right)
$$

In the presented equation above, the $\mathrm{A}$ is the number of all possible antenna subset of $\mathrm{N}_{\mathrm{s}}$ antennas out of $\mathrm{N}_{\mathrm{t}}$ antennas. All users select their subset that achieves the antenna selection criterion goal. Therefore, $\mathrm{L}=\mathrm{A}^{\mathrm{U}}$ number of possible subset that contain all possible transmit antenna amongst all users [7-8].

\subsection{Antenna Selection Based on All Users Jointly}

For this proposed strategy, antenna subset is selected from the overall users that satisfy the criteria for better performance of the systems. In this strategy of antenna selection, a subset of transmit antenna (NsxU) is selected out of $\left(\mathrm{N}_{\mathrm{t}} \mathrm{XU}\right)$, resulting in number of combination of subset 


$$
C=\left(\begin{array}{c}
N_{t} U \\
N_{S} U
\end{array}\right)
$$

\section{Simulation Results}

In this section, the bit error rate and sum rate performance of the antenna selection technique is presented for the MIMO-SC-FDMA-IDMA system. The maximum sum rate and minimum bit error rate criteria with bulk selection are considered in the simulation results.

The figure 2 ndemonstrates the combined sum rate performance of user 4 with four receive antennas and users 8 with eight receive antennas at the base station. The figure 3 presents the achievable sum rate performance with varying number of users. In both the figure of sum rate performance antenna selection at all users, jointly based strategy gives the better performance in comparison to each user selection or with no selection strategy.

The figure 4, 5, and 6 demonstrate the bit error rate performance of the system where each user has $\mathrm{Nt}=2$ transmit antenna and $\mathrm{Ns}=1$ antenna with both the antenna selection strategy being employed. In figure 4 and 5 , the number of receive antenna is eight at the base station and user count is 6 and 8 respectively, while in figure 6 , the comparison of bit error performance for user 4, 6, 8 and 10 with sixteen receive antenna has been presented employing both the overall antenna selection strategy and per user antenna selection strategy. From the simulation results presented in figure 4,5 , and 6 , it may be observed that as number of receive antenna increases, the antenna selection strategy, based on all user jointly, gives better performance due to increment in the diversity order. In figure 7 , it is presented that the bit error performances for user 4 and 8 , considering both the antenna selection strategy while figure 8 shows the bit error performance with varying count of users. In both all the simulations, the MIMO system with antenna strategy based on all user jointly demonstrate the better BER performance for the presented MIMO system.

Table 1. Parameters Used

\begin{tabular}{|l|l|}
\hline Parameter & Explanation/ Assumption \\
\hline Transmission bandwidth & $20 \mathrm{MHz}$ \\
\hline Allocated subcarriers & $256($ FFT size $=64)$ \\
\hline Subcarrier mapping & Interleaved \\
\hline Code block size & $1280 \mathrm{bits}$ \\
\hline Detection & MMSE \\
\hline
\end{tabular}

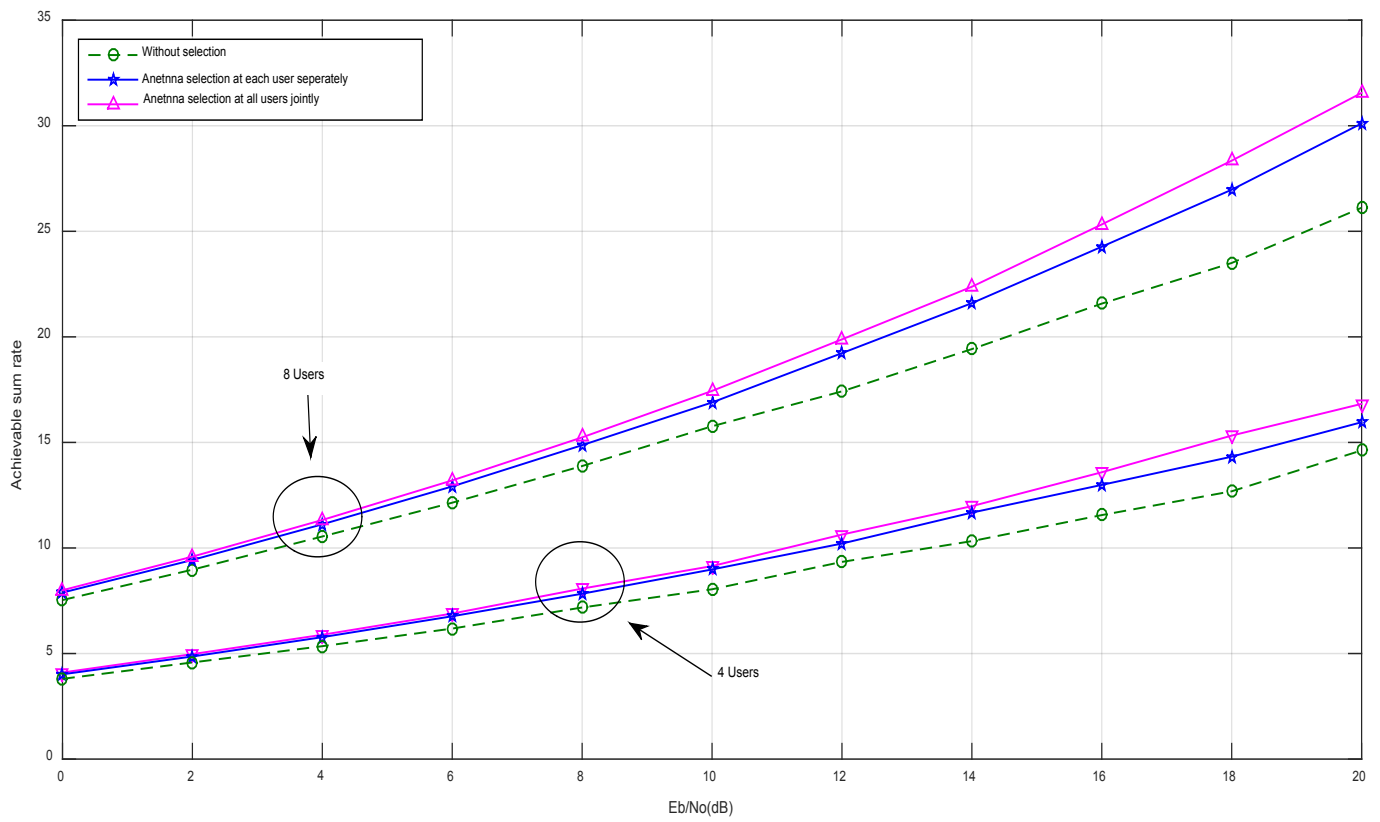

Figure 2. Sum Rate performance of both the transmit antenna selection strategies for $8 \times 8$ and $4 \times 4$ MIMO system 


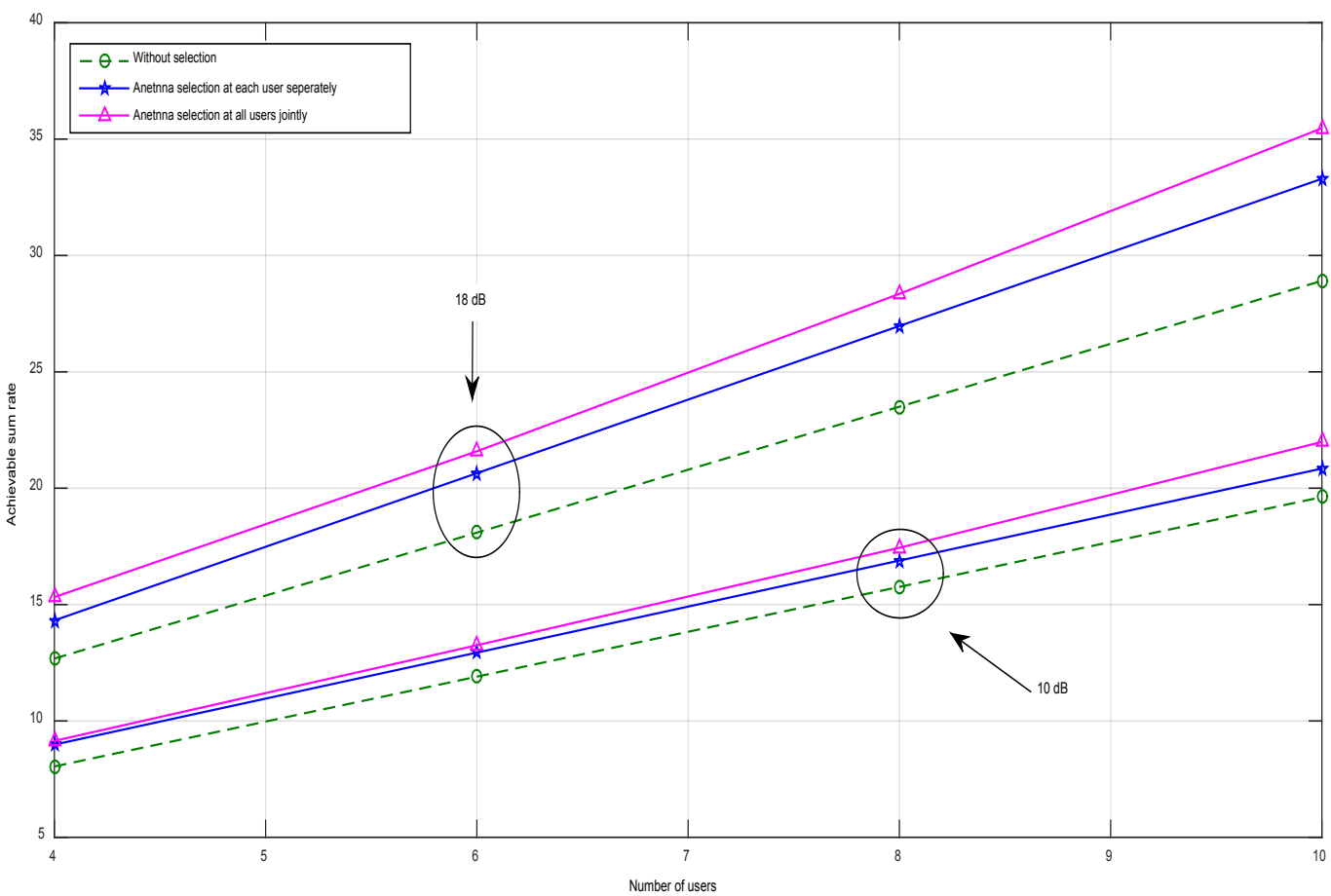

Figure 3. Achievable Sum rate Performance with Varying Number of Users $U$ at $10 \mathrm{db}$ and $18 \mathrm{dbEb} / \mathrm{No}$.

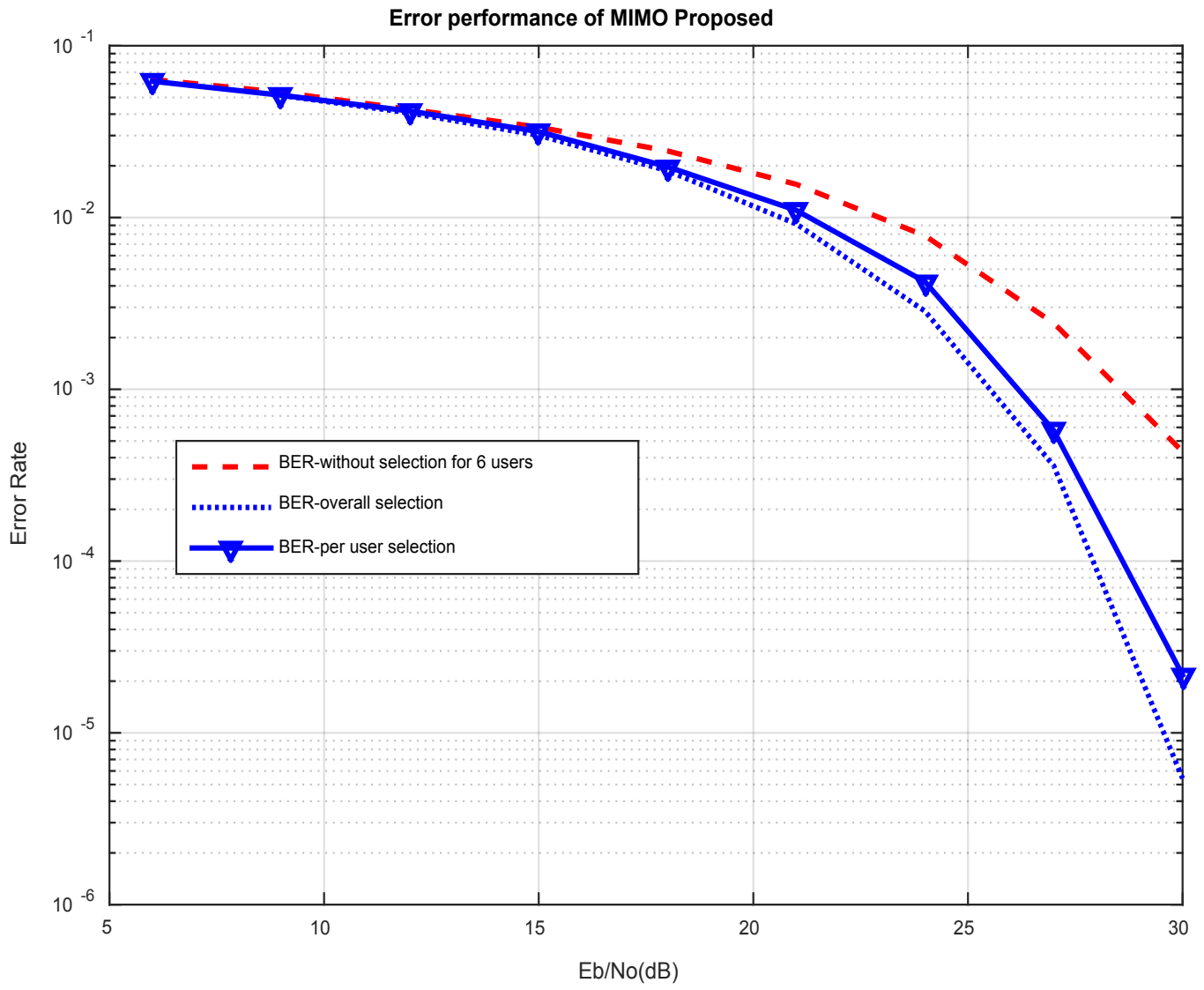

Figure 4. Bit Error Rate Performance of Antenna Selection Strategy for $6 \times 8$ MIMO System 


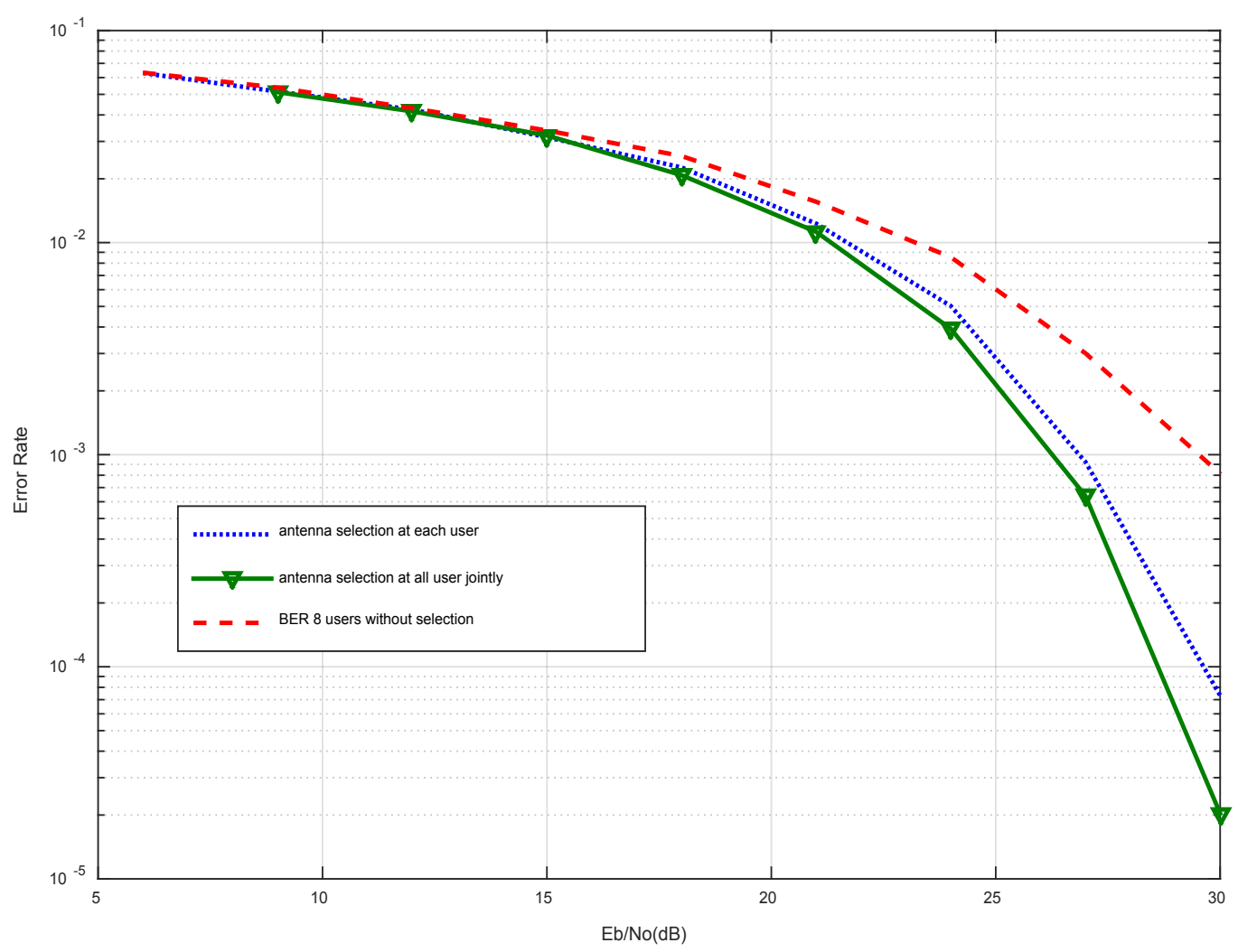

Figure 5. Bit Error Rate Performance of Antenna Selection Strategy for $8 \times 8$ MIMO Systems

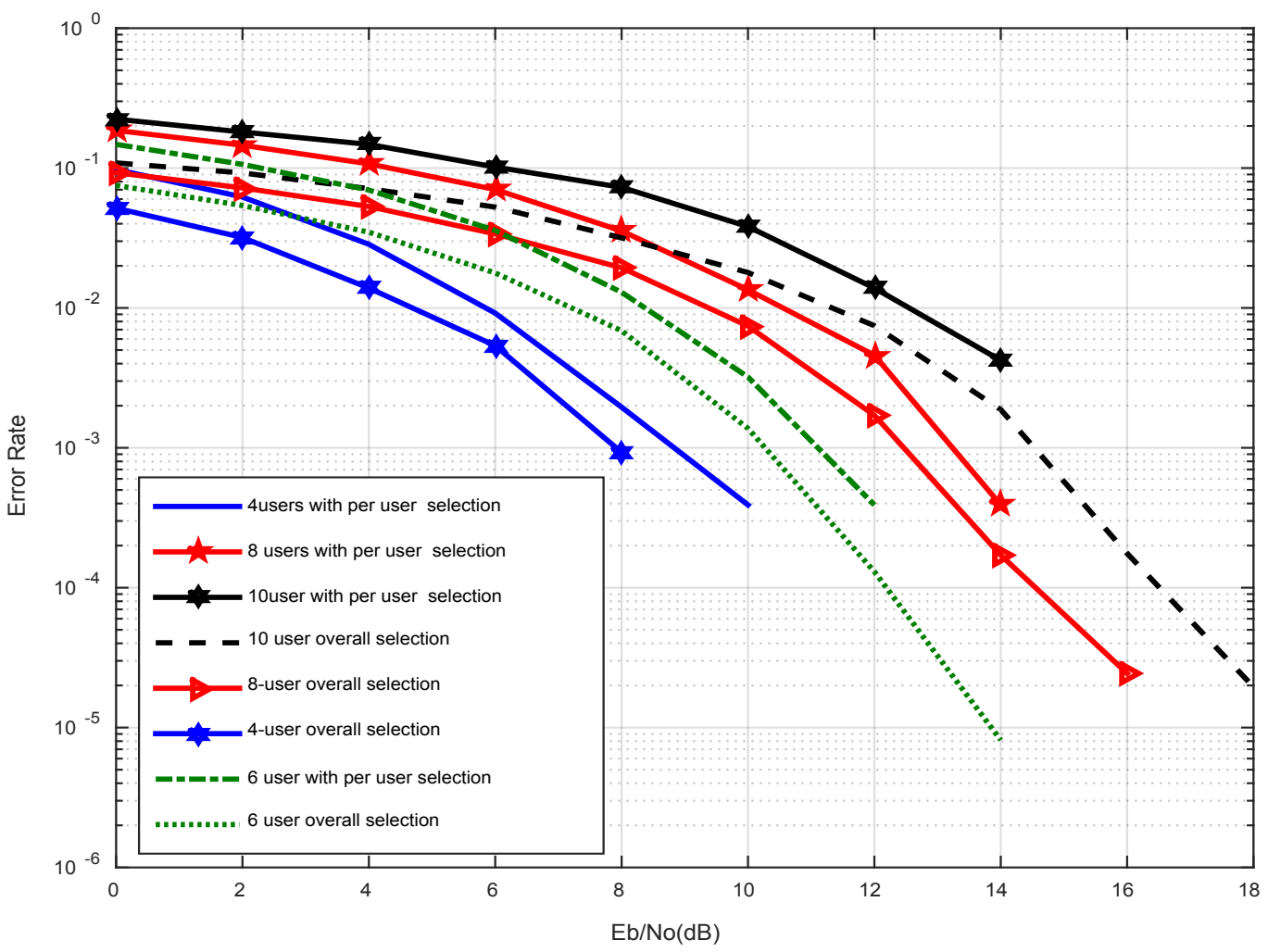

Figure 6. Comparison of Bit Error Rate Performance of Antenna Selection Strategy for user count 4, 6,8,10 with Nr=16 MIMO System 


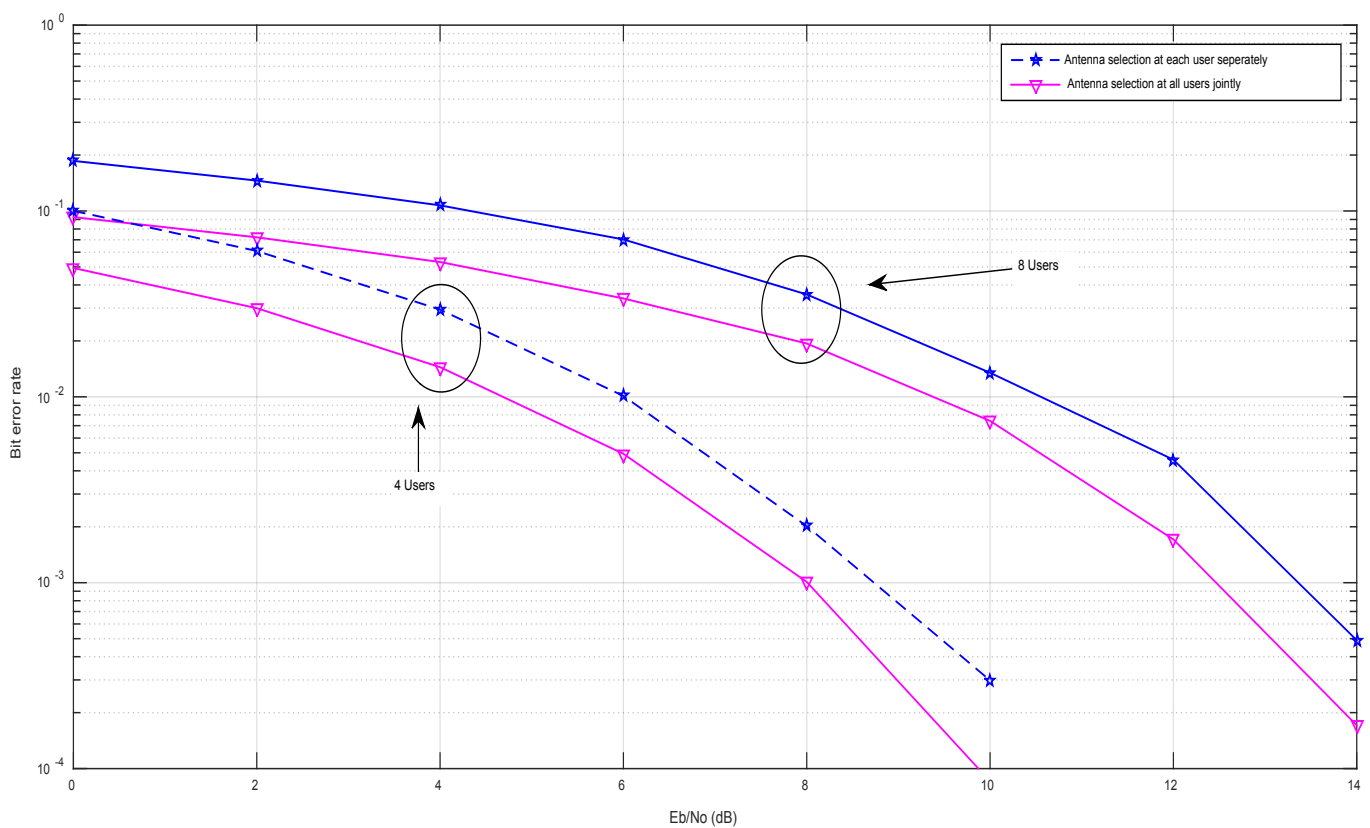

Figure 7. Comparison of Bit Error Rate Performance of Antenna Selection Strategy for User 4, and 8 with Nr=16 MIMO System

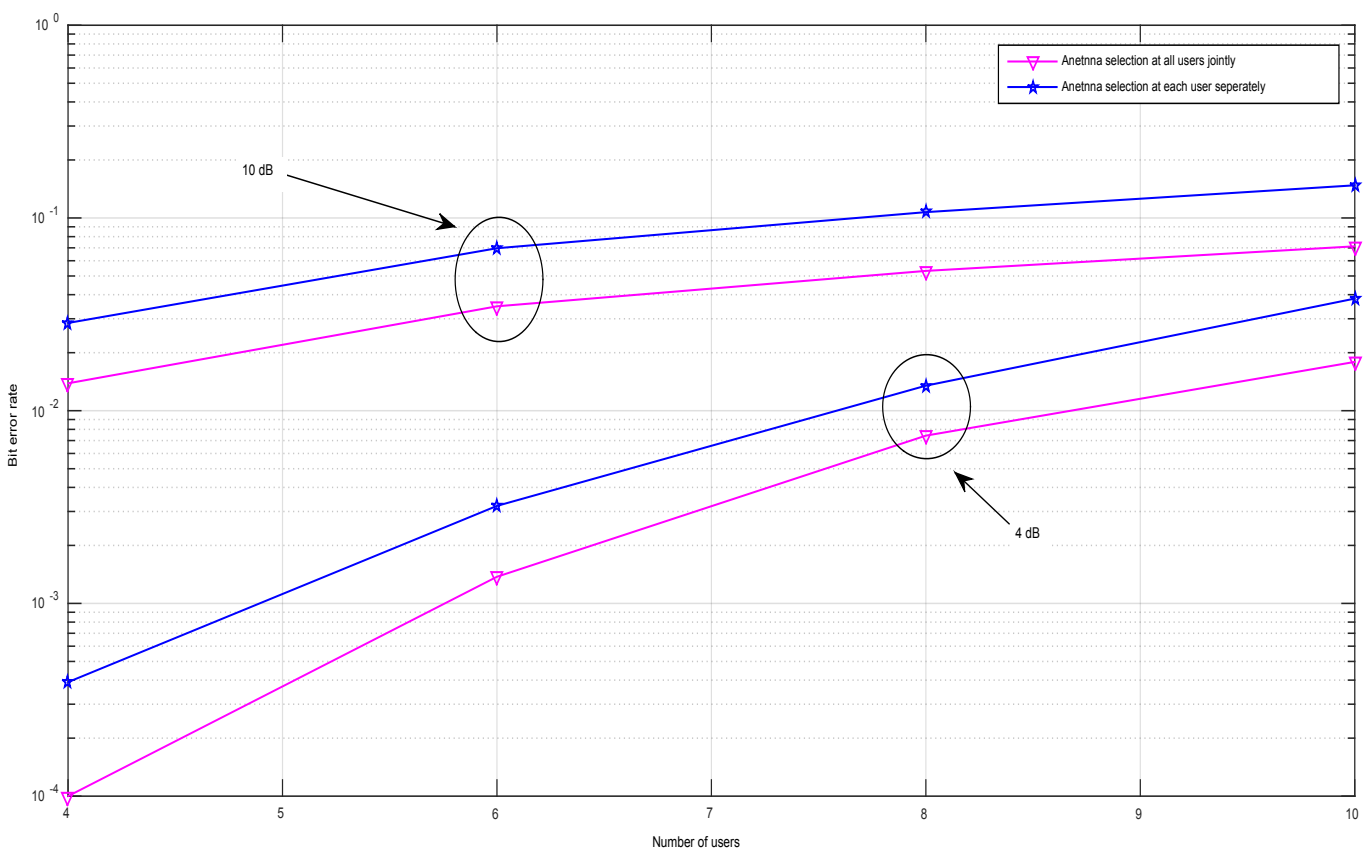

Figure 8. Comparison of Bit Error Rate Performance of Antenna Selection Strategy with Varying Number of Users

\section{Conclusions}

From the presented simulation results, it can be observed that the antenna selection strategy considering overall users, provides better sum rate and BER performance. At BER level of $10^{-3}$, the overall user selection strategy gives around $1 \mathrm{~dB}$ better performance in comparison to strategy of than the antenna selection at each user separately. However, it will be worth mentioning that strategy of considering overall users will increase a greater number of combinations of MIMO system making it more complex in terms of designing.

\section{REFERENCES}

[1] Z. Zhou, N. Ge, and X. Lin, "Reduced-complexity antenna selection schemes in spatial modulation," IEEE Communications Letters, Vol.18, No.1, pp.14-17, 2004.

[2] M.El-Absi, S. Galih, M. Hoffmann, M.El- Hadidy, and T. 
Kaiser, "Antenna selection for reliable MIMO-OFDM interference alignment systems: Measurement-based evaluation," IEEE Transactions on Vehicular Technology, Vol.65, No.5, pp.2965-2977, 2006.

[3] D. Gesbert, M. Shafi, D. Shiu, P.J. Smith, and A. Naguib, "From theory to practice: An overview of MIMO space-time coded wireless systems," IEEE J. Selected Areas in Commun., Vol.21, pp.281-302, April 2003

[4] S. Yang and L. Hanzo, "Fifty years of MIMO detection: The road to large-scale MIMOs," IEEE Communications Surveys \&Tutorials, Vol.17 No.4, pp.1941-1988, 2016.

[5] M. Agiwal, A. Roy and N. Saxena, "Next Generation 5G Wireless Networks: A Comprehensive Survey," in IEEE Communications Surveys \& Tutorials, Vol.18, no.3, pp.1617-1655, third quarter 2016.

[6] N.P. Le, F. Safaei, and L.C. Tran, "Antenna selection strategies for MIMO-OFDM wireless systems: An energy efficiency perspective," IEEE Transactions on Vehicular Technology, Vol.65 No.4, pp.2048-2062, 2016.

[7] Z. Lin, P. Xiao, B. Vucetic, and M. Sellathurai, “Analysis of receiver algorithms for LTE SC-FDMA based uplink MIMO systems," IEEE Transactions on Wireless Communications, Vol.9 No.1, pp.60-65,2010.

[8] M. Wu, B. Yin, G.Wang, C. Dick, J.R. Cavallaro, and C. Studer, "Large-scale MIMO detection for 3GPP LTE: Algorithms and FPGA Implementations," IEEE Journal of Selected Topics in Signal Processing, Vol.8, No.5, pp.916-
929, 2014.

[9] A. Kiayani, L. Anttila, Y. Zou, and M. Valkama, 'Channel estimation and equalization in multiuser uplink OFDMA and SC-FDMA systems under transmitter RF impairments', IEEE Transactions on Vehicular Technology, Vol.65 No.1, pp.82-99, 2016.

[10] L. Zhao, X. Sha, and X. Wu, "Achievable sum rate comparison of MIMO OFDMA and SC-FDMA systems with linear receivers," Proceeding of the IEEE $81^{\text {st }}$ Vehicular Technology Conference (VTC Spring), pp.1-5, 2015.

[11] C. Xu, Y. Hu, C. Liang, J. Ma and L. Ping, "Massive MIMO, Non-Orthogonal Multiple Access and Interleave Division Multiple Access," in IEEE Access, Vol.5, pp.14728-14748, 2017.

[12] C. Dong, K. Niu and J. Lin, "An Ordered Successive Interference Cancellation Detector with Soft Detection Feedback in IDMA Transmission," in IEEE Access, Vol.6, pp.8161-8172, 2018.

[13] Shukla M., Shukla A., Srivastava V.K., \& Tiwari S., "Performance Evaluation of MRC Diversity Scheme for Iterative IDMA Receivers", Proceedings of India Conference "INDICON-09", pp. 1-4, Gandhinagar India, 2009.

[14] Shukla M., Srivastava V.K. \& Tiwari S., "Analysis of Optimum Interleaver for Iterative Receivers in IDMA Scheme", Proceedings of International Conference on Computing and Networking "ICDCN 2009", Springer, pp. 400-407, 2009. 